\title{
The Effects of Combined Strength and Endurance Training on Running Performance the Following Day
}

\author{
Kenji Doma ${ }^{1}$ and Glen Bede Deakin ${ }^{2}$ \\ ${ }^{1} 1$ James Cook Drive, Rehab Sciences Bdng (DB-43), Institute of Sport and Exercise Science \\ James Cook University, Douglas Campus, Townsville QLD 4811, AUSTRALIA \\ Email:kenji.doma@jcu.edu.au \\ 214-88 McGregor Road, Institute of Sport \& Exercise Science \\ James Cook University, Cairns Campus, QLD 4870, AUSTRALIA \\ [Received September 4, 2012; Accepted February 5, 2013; Published online February 22, 2013]
}

\begin{abstract}
This study examined running economy (RE) during a two-stage incremental protocol that was combined into an endurance training session 6 hours following a strength training session. In addition, this study investigated running performance which consisted of a two-stage RE protocol and time to exhaustion (TTE) the day after strength and endurance training sessions undertaken on the same day. Twelve trained and moderately trained male runners performed strength and endurance training sessions 6 hours apart with running performance tests conducted the following day. Cost of running $\left(C_{R}\right)$ and rate of perceived exertion (RPE) were collected during the endurance training session whereas $C_{R}$, RPE and TTE were collected during the running performance test. Maximal voluntary contraction (MVC) tests were conducted prior to and following the strength and endurance training sessions and the running performance tests. The results showed that $C_{R}$ significantly increased during the second stage of the endurance training session $(p<0.05)$. During the running performance test, significant increases were found for $C_{R}$ during the first and second stages and for RPE during the second and third stages $(p<0.05)$. The MVC was significantly reduced immediately following the strength training sessions, pre-post the endurance training session, and running performance test $(p<0.05)$. The findings in the current study show that RE is impaired 6 hours following a strength training session. Furthermore, combined strength and endurance training on the same day appears to cause an accumulation effect of fatigue which impairs running performance the following day.
\end{abstract}

Keywords: running economy, time to exhaustion, kinematics, muscle force generation capacity

\section{Introduction}

Combining strength and endurance training sessions in the one training program, referred to as concurrent training (Hickson, 1980), has been shown to interfere with strength development (Leveritt et al., 1999). This interference has been suggested to occur due to preceding endurance exercises compromising optimal force production during subsequent strength training sessions, known as the "acute hypothesis" (Leveritt et al., 1999). Indeed, reports have shown that endurance training impairs maximal voluntary contraction (MVC) for 6 hours post training (Bentley et al., 2000), indicating that endurance exercises may cause detrimental effects on strength training performance. However, studies have also shown that strength training reduces MVC for over 48 hours (Brentano and Kruel, 2011; Hakkinen et al., 1988), suggesting that endurance performance may be impaired in the hours following a strength training session. Subsequently, chronic endurance development could be compromised if the quality of each endurance training session is interfered due to preceding strength training sessions. Examining the acute effects of strength training (e.g. several hours post training) on endurance performance may shed light on factors attributing to the possible interference of chronic endurance development during concurrent training.

Studies have reported that strength training impaired running economy (RE) 8 hours post (Palmer and Sleivert, 2001) and compromised running time- 
trial performance (Marcora and Bosio, 2007) and repeated sprint ability (Twist and Eston, 2005) 24 hours post. In contrast, studies have shown no effect on RE 24 hours following strength training despite increases in creatine kinase and muscle soreness (Marcora and Bosio, 2007; Paschalis et al., 2007). Collectively, these findings suggest that strength training may impair sub-maximal endurance performance on the same day yet cause no affect the following day, or affect maximal endurance performance the following day. However, these studies (Marcora and Bosio, 2007; Paschalis, et al., 2007; Twist and Eston, 2005) collected endurance performance measures following a single training session. To date, the effect of combining the two modes of exercises on the same day and their effect on various performance measures the following day remains unclear.

Studies have reported that MVC was compromised 6 hours post endurance training (Bentley et al., 2000) and 24 hours post strength training. Therefore, it is presumable that performing strength and endurance training sessions 6 hours apart on the same day will impair various performance measures the following day. However, sub-maximal endurance exercises have been shown to accelerate recovery following strenuous exercises due to an increase in blood flow (Faude et al., 2009). Subsequently, an endurance training session performed after a strength training session with several hours of recovery may function as a buffer and limit the impact of fatigue on endurance performance the following day. Furthermore, an overnight passive recovery period (e.g. 16-24 hours) may provide sufficient recovery from performing strength and endurance training sessions on the same day. This has been highlighted by previous studies where full recovery from high intensity endurance exercises occurred within 24 hours (Bentley, et al., 2000) and that no deletrious effects on sub-maximal running performance was found 24 hours following strength training (Marcora and Bosio, 2007).

The purpose of the current study was two fold. First, to examine the acute effect of a strength training session on various performance measures (i.e. MVC, RE, running time-to-exhaustion and kinematics) performed 6 hours later. Second, examine the combined effect of performing strength and endurance training sessions on the same day on performance measures the following day. It was hypothesised that attenuation in running performance will occur 6 hours following strength training as well as the following day as a result of undertaking both strength and endurance training sessions on the same day.

\section{Methods}

\subsection{Participants}

A group of 12 trained and moderately trained male runners (mean \pm standard deviation: age 23.4 $\pm 6.4 \mathrm{yrs}$; height $1.8 \pm 0.1 \mathrm{~m}$; body mass $75.0 \pm 8.2$ $\mathrm{kg}$; maximal oxygen uptake $\left(\mathrm{VO}_{2 \max }\right) 62.5 \pm 6.0$ $\mathrm{mL} \cdot \mathrm{kg}^{-1} \cdot \mathrm{min}^{-1}$ ) who had not undertaken lower extremity strength training exercises for two months took part in the study. The trained runners were middle to long distance runners (1500-10,000 m) covering at least $50 \mathrm{~km}$ per week and had all run a $10,000 \mathrm{~m}$ time trial within 35 minutes during the last 6 months. The moderately endurance trained runners were covering $5-10 \mathrm{~km}$ per week in addition to their various sporting backgrounds. Each participant completed informed consent before taking part in any testing procedures. All procedures in this study were approved by the Institutional Human Research Ethics Committee and were run according to the Declaration of Helsinki.

\subsection{Research design}

The study was conducted across 5 weeks. The first four weeks were used as preparation and the experiments were conducted during the fifth week (Figure 1). During the first week, a familiarisation session and a $\mathrm{VO}_{2 \max }$ test were conducted. In addition to familiarity purposes, a six repetition maximum (6RM) assessment was conducted according to previous guidelines (Baechle and Earle, 2008) during the familiarisation session. The $\mathrm{VO}_{2 \max }$ test was a continuous incremental running protocol to exhaustion on a treadmill as described previously (Doma et al., 2012b). During the second week, two running economy (RE) tests were carried out for familiarity. The data collected during the second RE test were used as baseline (Base-RE). Two strength sessions were undertaken during the third and fourth week as a washout period since a second strength training session has been shown to limit the negative impact a single strength training session has on running per- 


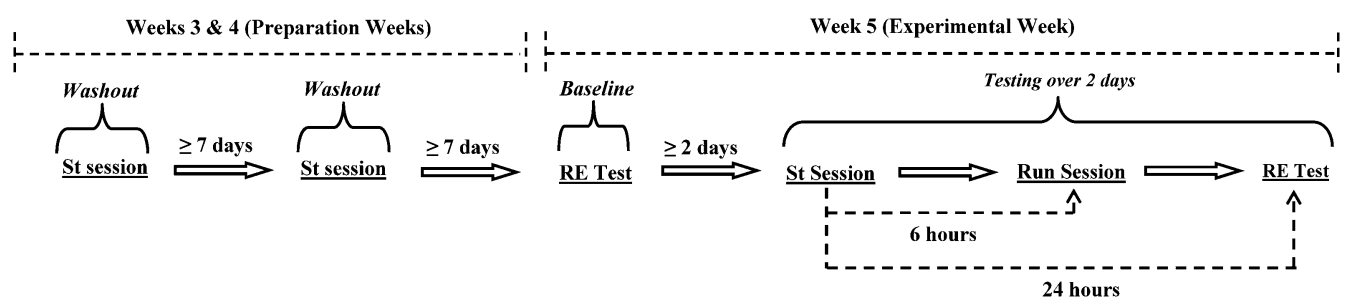

Figure 1 Schematic of the research design

formance due to repeated bout effects (Burt et al., 2012). During the strength training session, exercises were performed in the order of leg press (Maxim MF701, Australia) for 6 sets and leg curls and leg extensions (Avanti, B253 Olympic Bench, Australia) for four sets at 6RM, respectively, with three minutes of rest in between each set and exercise. In the last week, a strength session and a running session were conducted 6 hours apart with a RE test (Post-RE test) 24 hours following the strength session. The running session was treated as an endurance training session which was separate from the $\mathrm{RE}$ test in order to investigate the accumulation effect of strength and endurance training on running performance (i.e. RE test) over two consecutive days. All RE tests were conducted at the same time of the day. Maximal voluntary contraction (MVC) was also measured pre-post the strength session, running session, and Post-RE test.

\subsubsection{Running session}

Running sessions were performed following a progressive warm-up by walking on the treadmill for 5 minutes then jogging at $8-, 10$ - and $12 \mathrm{~km} \cdot \mathrm{hr}^{-1}$ for one minute, respectively. The running session consisted of three incremental stages with intensities set at $70-, 90-$ and $110 \%$ of the second ventilatory threshold $\left(\mathrm{VT}_{2}\right)$ and two minutes of rest between each stage. The first two stages consisted of two 10 minute stages with four 2 minute intervals during the last stage with a work to rest ratio of $1: 1$. During the first two stages, oxygen cost of running $\left(C_{R}\right)$, RPE and lower extremity kinematics were collected. The $\mathrm{C}_{\mathrm{R}}$ was calculated such that $\mathrm{VO}_{2}$ was expressed relative to body mass to the power of 0.75 per metre $\left(\mathrm{mL} \cdot \mathrm{kg}^{-0.75} \cdot \mathrm{m}^{-1}\right)$ as this has been shown to minimize inter-individual variability (Doma et al., $2012 \mathrm{~b}$ ). In addition, $C_{R}$ was averaged during the last 5 minutes of the first two stages to ensure that the participants reached steady-state running (Doma et al., 2012b). Steady-state was defined when the change in $\mathrm{VO}_{2}$ was $<10 \%$ per minute (Reeves et al., 2004). The $\mathrm{VT}_{2}$ was determined from the $\mathrm{VO}_{2 \max }$ test by ascertaining the inflection point of ventilation with respect to carbon dioxide production on a scatter diagram (Neder and Stein, 2006). The RPE was collected during the $9^{\text {th }}$ minute of the first two stages.

\subsubsection{Running economy test}

The Base-RE and Post-RE tests were conducted following a warm-up identical to the running session. The RE tests consisted of three incremental stages running at $70-, 90-$, and $110 \%$ of VT2, respectively (Doma et al., 2012b). There were two minutes of recovery between the three stages. The $C_{R}$, RPE and lower extremity kinematics were collected during the first two stages identical to that during the running sessions. However, whilst the last stage was treated as an interval period for the running session, the participants ran to exhaustion during the RE test to determine time to exhaustion (TTE). RPE was collected every minute during TTE and the RPE of the middle time points during the shortest TTE of a given RE test was used for comparisons (e.g. if TTE for a given participant was 5 minutes for Post-RE, then the RPE for the third minute of Post-RE was compared with the third minute of Base-RE). Peak $C_{R}$ was also collected during TTE. The first two stages of the running session and RE tests were standardised to compare running performance variables between the Base-RE test, running session and Post$\mathrm{RE}$ test whilst allowing the running session to contribute as an endurance training session.

\subsubsection{Kinematic Analyses}

Running gait was captured at 9 minutes $30 \mathrm{sec}-$ onds of the first two stages of Base-RE, running session and Post-RE test. At least 10 strides of kinematic data were recorded for each motion capture at $100 \mathrm{~Hz}$ using a three-dimensional 8-camera optical motion analysis system (VICON Motion Systems, Oxford, UK). The optical cameras were stati- 
cally calibrated for each testing session and ensured an image error of $<0.15$ pixels. The measuring volume covered $1.5 \mathrm{~m} \times 3 \mathrm{~m} \times 2 \mathrm{~m}$ (width, length, height). The pelvis, thighs, shank and the feet were captured using 16 retro-reflective markers $(14 \mathrm{~mm}$ diameter) placed by a single well trained investigator according to Nexus Plug-in Gait Model. Running gait parameters included ankle range of motion $\left(\mathrm{A}_{\mathrm{ROM}}\right)$, maximum knee flexion during swing $\left(\mathrm{KF}_{\mathrm{S}}\right)$, maximum knee flexion after foot strike $\left(\mathrm{KF}_{\mathrm{AS}}\right)$ and hip range of motion $\left(\mathrm{H}_{\mathrm{ROM}}\right)$ in the sagittal plane. Kinematic parameters were limited to 2-dimensions as the reliability of lower extremity kinematics has been reported to be questionable in the transverse and frontal planes (Doma et al., 2012a). Raw kinematic data were filtered using Woltring filtering routing with the mean squared error at $20 \mathrm{~mm}^{2}$ based on residual analyses (Winter, 2008).

\subsubsection{Maximal voluntary contraction test}

A custom-built dynamometer chair (James Cook University, Australia) was used to conduct MVC tests. Three maximal isometric contractions of the knee extensor muscles were performed for 6 seconds with 1.5 minutes rest between each contraction (Doma and Deakin, 2012) whilst the knee joint was positioned at $110^{\circ}$. Torque was calculated by averaging the values over the 6-second contraction with the largest torque being reported amongst the three contractions.

\subsubsection{Statistical analysis}

All data are expressed as mean \pm standard deviation. A one-way analysis of variance was used to determine differences in $C_{R}$, RPE and lower extremity kinematics (i.e. $\mathrm{A}_{\mathrm{ROM}}, \mathrm{KF}_{\mathrm{S}}, \mathrm{KF}_{\mathrm{AS}}$ and $\mathrm{H}_{\mathrm{ROM}}$ ) between the Base-RE test, running session and Post$\mathrm{RE}$ test and to analyse differences in torque between the six different time points (i.e. prior to and following the strength session, running session and PostRE test) of the MVC tests and pairwise comparisons with Bonferroni's adjustments. Paired T-tests were conducted to analyse the difference in TTE between Base-RE and Post-RE tests. Pearson's product moment correlation was used to analyse the relationship between the running performance variables (i.e. $\mathrm{C}_{\mathrm{R}}$, RPE and TTE), running kinematics and torque. Data analyses were conducted using the Statistical Package for Social Sciences (SPSS, version 18, Chicago, IL) with the alpha level at 0.05 .

\subsubsection{Sample size}

Based on an in-house reliability study of the RE test and MVC test used in the current study, the within-subject coefficient of variation $(\mathrm{CV})$ for $\mathrm{C}_{R}$, RPE, TTE and torque production amongst trained and moderately endurance trained men $(n=14)$ were 2.5-, 3.6-, 9.2- and 8.3\%, respectively (Doma et al., $2012 \mathrm{~b}$ ). With the use of the estimation of measurement error based on a nomogram and CV (statistical power of 90\%) (Atkinson and Nevill, 2006), the percentage worthwhile differences for the current sample size $(n=12)$ for $C_{R}$, RPE, TTE and torque production were found to be 3.9-, 5.5-, 13.6- and $12.3 \%$, respectively. These worthwhile differences are smaller than previous studies that have shown significant differences in RE (Palmer and Sleivert, 2001), RPE (Doma et al., 2012), TTE (Doma et al., 2012) and torque production (Palmer and Sleivert, 2001) as a result of a particular strength training session.

\section{Results}

\subsection{Running performance}

The $C_{R}$ was significantly greater for the Post-RE test compared to the Base-RE test during stages 1 and $2(p<0.05)$ and significantly greater for the running session compared to the Base-RE test during stage $2(p<0.05$; Figure 2$)$. The RPE was significantly greater for Post-RE test compared to Base-RE test $(p<0.05$; Figure 2$)$ during stage 2 although TTE was significantly less during Post-RE $(269 \pm 69 \mathrm{sec})$ compared to Base-RE $(331 \pm 100 \mathrm{sec})$ $(p<0.01)$.

\subsection{Running kinematics}

The $\mathrm{H}_{\mathrm{ROM}}$ was significantly less for the running session and Post-RE test compared to Base-RE test $(p<0.05)$ during stages 1 and 2 (Figure 3). The $\mathrm{KF}_{\mathrm{S}}$ for Post-RE test was significantly less than Base-RE test $(p<0.05)$ during stage 2 . The torque was significantly reduced for all time points following strength training (Figure 4).

\subsection{Relationship between performance variables}

No significant relationships were found between the running performance variables (i.e. $C_{R}$, RPE 

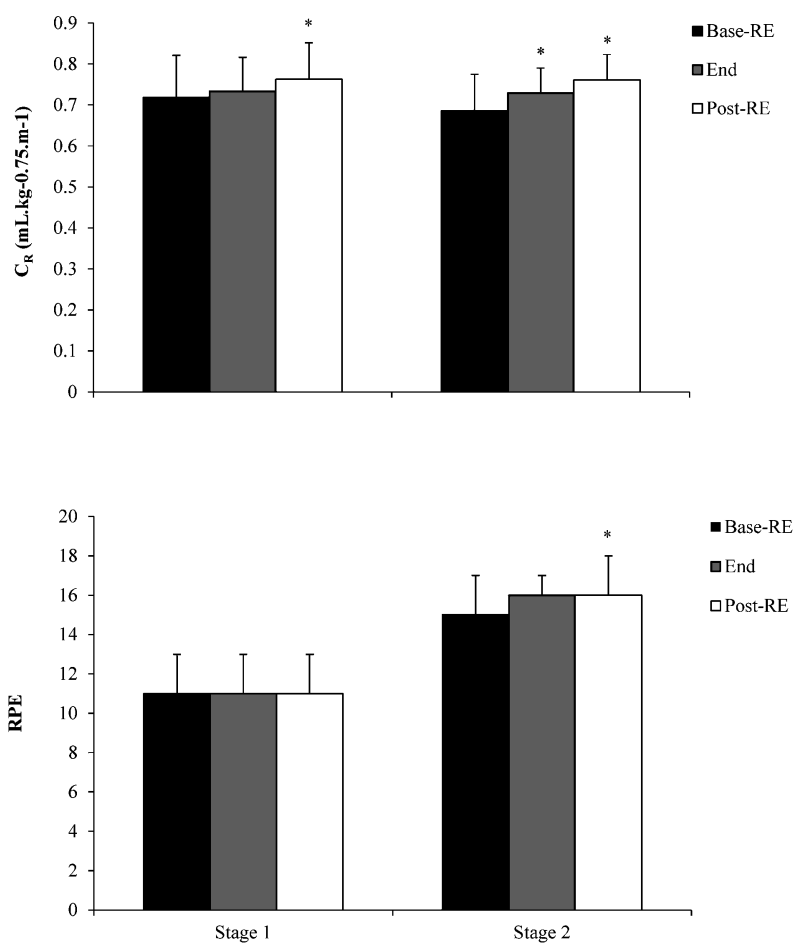

Figure 2 The oxygen cost of running (CR) and rate of perceived exertion (RPE) during the stages 1 and 2 of the base running economy test (Base-RE), running session (End) and post running economy test (Post-RE).

*Significantly greater than the base running economy test $(\mathrm{P}<0.05)$

and TTE), running kinematics and torque for the percentage differences between Base-RE, running session and Post-RE during Stages $1(r=0.12-0.41)$, $2(r=0.03-0.51)$ and TTE $(r=0.06-0.18)(p>0.05)$.

\section{Discussion}

The results showed that strength training increased $C_{R}$ and reduced $H_{R O M}$ and torque 6 hours post. In addition, $\mathrm{C}_{\mathrm{R}}$ increased and $\mathrm{H}_{\mathrm{ROM}}, \mathrm{KF}_{\mathrm{S}}$ and torque decreased one day following strength and endurance training performed 6 hours apart. These findings support the hypothesis that running performance will be impaired 6 hours following strength training and that the combination of strength and endurance training performed on a single day will affect running performance the following day.

\subsection{Impact of strength training on running perfor- mance on the same day}

The increase in $C_{R}$ during the running sessions is similar to a previous study that has shown an eleva-
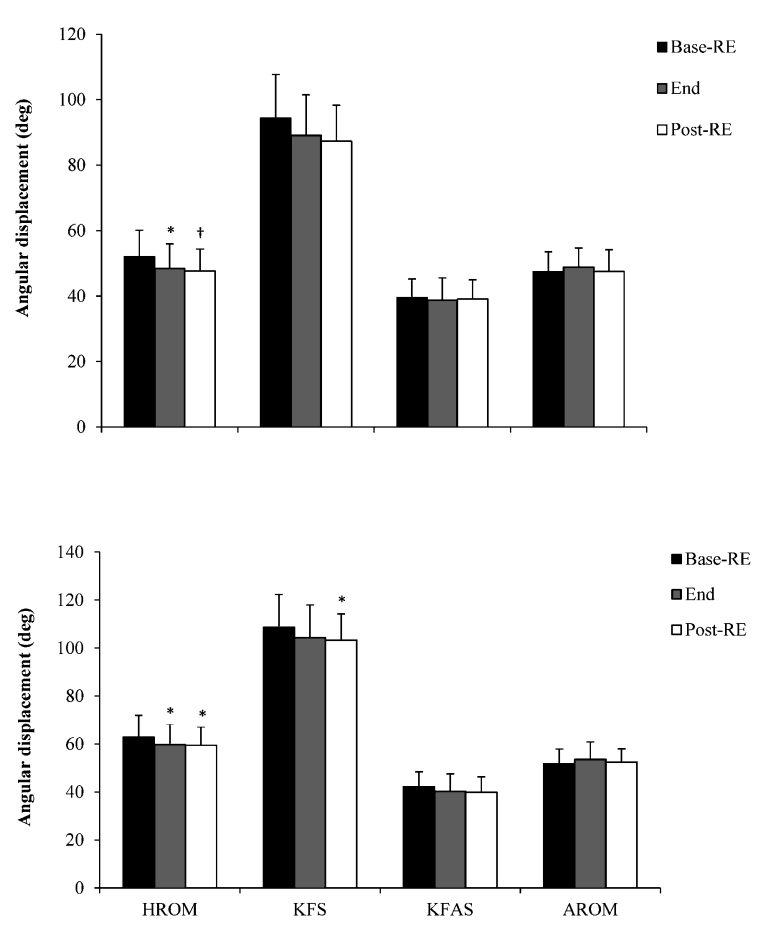

Figure 3 The angular displacements in degrees (deg) for hip range of motion $\left(\mathrm{H}_{\mathrm{ROM}}\right)$, knee flexion during the swing phase $\left(\mathrm{KF}_{\mathrm{S}}\right)$, knee flexion after foot strike $\left(\mathrm{KF}_{\mathrm{AS}}\right)$ and ankle range of motion $\left(\mathrm{A}_{\mathrm{ROM}}\right)$ during Stages 1 (top) and 2 (bottom) for the base running economy test (Base-RE), running session (END) and post running economy tests (Post-RE).

*Significantly less than the base running economy test $(\mathrm{P}<0.05) ; \uparrow(\mathrm{P}<0.01)$

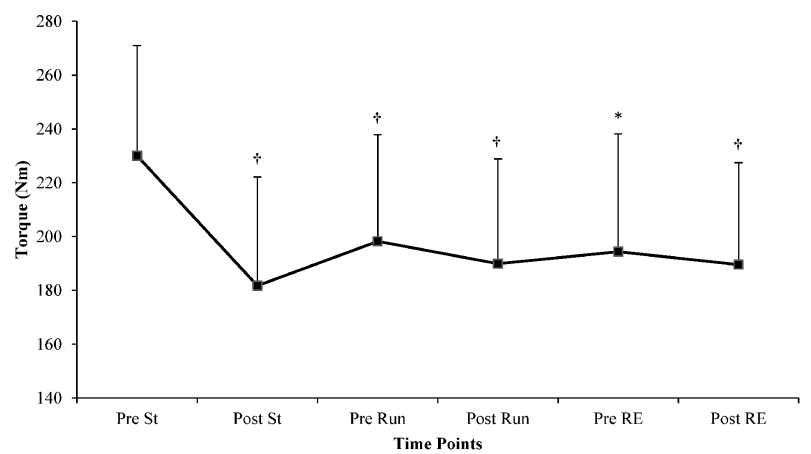

Figure 4 The torque production measure prior to- (Pre St) and following (Post St) the strength training session, prior to- (Pre Run) and following (Post Run) the running session and prior to(Pre RE) and following (Post RE) the experimental running economy test.

*Significantly less than Pre St $(\mathrm{P}<0.05) ; \uparrow(\mathrm{P}<0.01)$

tion in $\mathrm{VO}_{2}$ during sub-maximal running 8 hours following strength training (Palmer and Sleivert, 2001). A recent study by Doma and Deakin (2012) reported no effect on RE 6 hours following strength training. However, the authors suggested that the deleterious effects of strength training on RE was limited since 
strength training exercises were performed with slow eccentric contractions (i.e. 4 seconds). Alterations in $C_{R}$ may have been observed in the present study since the strength training exercises were performed at faster contraction velocities (i.e. self-paced). It has previously been reported that strength training with fast compared to slow contraction velocities caused greater exercise-induced fatigue (Ide et al., 2011). However, further research is warranted to systematically examine the effect of strength training contraction velocity on running performance.

The current study showed a significant reduction in $\mathrm{H}_{\mathrm{ROM}} 6$ hours following strength training and agrees with previous studies that have shown a compromise in lower extremity joint range of motion during running as a result of exercise-induced fatigue (Paschalis, et al., 2007; Reeves et al., 2003). The reduction in $\mathrm{H}_{\mathrm{ROM}}$ following strength training in the present study may be due to increased muscle stiffness as there is evidence suggesting that exhaustive exercises alter the visco-elastic properties of the tendons and increase the stiffness of the joint, muscles and tendons (Reeves, et al., 2003). As a result, the reduction in joint range of motion may alter the length-tension relationship and impair neuromuscular performance of the lower extremity. In fact, Kellis and Liassou (2009) showed that localised muscle fatigue following isokinetic contractions caused changes in lower extremity kinematics in conjunction with increased muscle activation of the knee extensors and plantar flexors during toe-off and increased knee flexors during the swing phase. The increase in muscle activity suggests that muscles may use additional energy to compensate for alterations in running kinematics which would elevate the metabolic cost of running.

\subsection{Impact of strength and endurance training on running performance the following day}

The findings from the present study showed further alterations in $C_{R}$ and lower extremity running kinematics during Post-RE (i.e. one day following strength and endurance training) compared to the running session (i.e. 6 hours following strength training). The $C_{R}$ during the Post-RE test was significantly greater at stages 1 and 2 whereas the $C_{R}$ was significantly greater only at stage 2 during the running session. In addition, a significant increase in RPE was only found during the Post-RE test at stage 2. Furthermore, whereas $\mathrm{H}_{\mathrm{ROM}}$ was the only kinematic parameter that was significantly less during the running session, both $\mathrm{H}_{\mathrm{ROM}}$ and $\mathrm{KF}_{\mathrm{S}}$ were significantly less during the Post-RE test. These findings demonstrate that strength and endurance training sessions performed in a single day, despite a 6 hour recovery period, generates an accumulation effect of fatigue on running performance the following day. Whilst TTE was not collected during the running session as it was treated as an endurance training session, TTE was significantly reduced during Post-RE which exemplifies that concurrent strength and endurance training also impairs running performance at maximal effort the following day.

Whilst previous studies have found that sub-maximal endurance sessions may accelerate recovery (Faude, et al., 2009), the current study showed an increase in $C_{R}$ during Post-RE the following day. Such findings suggest that the running session performed in conjunction with strength training may have induced an additional stimulus of fatigue as opposed to facilitating recovery the following day. Drummond et al (2005) examined strength and endurance training sequence on excess post exercise oxygen consumption (EPOC). The authors showed that EPOC was greatest when strength training preceded endurance training compared to strength training alone, endurance training alone or when endurance preceded strength training. These findings indicate that a combination of strength and endurance training elevates metabolic cost and that strength training may be the primary contributor to the accumulation effect of the two modes of training. Given the nature of the current findings, it would be interesting to systematically examine the effect of sequence of strength and endurance training on running performance the following day.

\subsection{Relationship between performance variables}

Whilst the present findings showed an increase in $\mathrm{C}_{\mathrm{R}}$ with a concomitant reduction in muscle force generation capacity (MFGC) 6 hours following strength training, there was no relationship between the percentage differences in MFGC and RE. These results are similar to findings by Chen and colleagues (2009) where RE was impaired for only two days following downhill running yet MFGC was reduced for five days. The lack of relationship between RE and 
MFGC suggests that the compromise in RE may not only be governed by MFGC. This is not surprising given that attenuation in RE following strenuous exercises have been associated with muscle soreness, muscle damage, increased level of perceived exertion and alterations in running kinematics (Chen, et al., 2009; Paschalis, et al., 2007). Thus, the mechanisms responsible for a decrement in $\mathrm{RE}$ appear to be a complex phenomenon involving various physiological and physical measures. Nonetheless, given that $C_{R}$ was increased with a concomitant reduction in torque, MFGC may in part have contributed to alterations in $C_{R}$ following strength training in the present study.

No significant relationships were found between changes in lower extremity kinematics, MVC and running performance variables (i.e. $C_{R}$, RPE and TTE) from the current results. These findings are in agreement with Collins et al. (2000) who reported no significant relationship between RE and lower extremity running kinematics following high intensity interval training. The lack of relationship between running performance variables and running kinematics could account for differences in coping strategies given that the training background varied between the participants in the present study. Studies have found significant day-to-day variability in step length within individuals (Morgan et al., 1991). It has also been shown that variability and fluctuation in running gait cycle were greater for non-runners compared to experienced runners, demonstrating that spatiotemporal organisation during running differs between individuals with varying running experiences. Subsequently, whilst a relationship was not found between running kinematics and the running performance variables, given that significant differences were found in both performance measures following strength and endurance training, it is reasonable to assume that changes in running technique may in part have contributed to an increase in $\mathrm{C}_{\mathrm{R}}$ in the current study. However, this speculation warrants further investigation by incorporating a more homogenous group of runners.

\section{Conclusion}

This study showed that strength training elevates $C_{R}$, decreases MFGC and reduces lower extremity joint range of motion with 6 hours of recovery. Additionally, performing strength and endurance train- ing on the same day appears to intensify the effects of strength training on running performance the following day compared to strength training alone. Whilst studies have shown that strength training does not affect RE despite exercise-induced muscle damage with a 24 hour recovery period (Marcora and Bosio, 2007; Paschalis, et al., 2007), the current findings indicate that strength training may cause detrimental effects on sub-maximal and maximal running performance 24 hours post when combined with a moderate to high intensity endurance training session.

\section{Practical application}

According to the current findings, high intensity strength training impaired RE 6 hours post and impaired RE and TTE 18 hours following strength and endurance training sessions performed on the same day 6 hours apart. Subsequently, moderate to high intensity running sessions should not be performed within the hours following high intensity strength training as prescribed in the current study amongst moderately trained to trained endurance athletes with minimal strength training experience or those that are detrained from strength training. When incorporating high intensity strength and endurance training into a concurrent training program, the two modes of training sessions should be prescribed on alternating days in order to ensure recovery between each mode of training.

\section{Acknowledgements}

The authors would like to thank Ms. Laura Webb, Ms. Samantha Abraham, Mr. Aaron Hynds, Mr. Blair Hunt and Mr. Keiran Kowalski for their assistance with data collection. The authors would also like to thank Mrs. Marian Dohma for her editing of this manuscript.

\section{References}

Atkinson, G., and Nevill, A. M. (2006). Sport and Exercise Physiology Testing Guidelines: Sport Testing Volume 1 (3rd ed.). Routledge: London.

Baechle, T. R., and Earle, R. W. (2008). Essentials of strength training and conditioning (3rd ed.). Champaign, IL: Human Kinetics.

Bentley, D. J., Smith, P. A., Davie, A. J., and Zhou, S. (2000). Muscle activation of the knee extensors following high intensity endurance exercise in cyclists. Eur. J. Appl. Physiol., 81: 297-302.

Brentano, M. A., and Martins Kruel, L. F. (2011). A review on strength exercise-induced muscle damage: applications, adaptation mechanisms and limitations. J. Sports Med. Phys. Fitness., 51: 1-10. 
Burt, D., Lamb, K., Nicholas, C., and Twist, C. (2011). Effects of repeated bouts of squatting exercise on sub-maximal running performance. Eur. J. App. Physiol. Epub ahead of print.

Chen, T. C., Nosaka, K., Lin, M. J., Chen, H. L., and Wu, C. J. (2009). Changes in running economy at different intensities following downhill running. J. Sports Sci., 27: 1137-1144.

Collins, M. H., Pearsall, D. J., Zavorsky, G. S., Bateni, H., Turcotte, R. A., and Montgomery, D. L. (2000). Acute effects of intense interval training on running mechanics. J. Sports Sci., 18: 83-90.

Doma, K., and Deakin, G. B. (2012). The acute effects of intensity- and volume- of strength training on running performance. Eur. J. Sport Sci., In Press.

Doma, K., Deakin, G. B., and Sealey, R. M. (2012a). The reliability of lower extremity and thoracic kinematics at various running speeds. Int. J. Sports Med., 33: 364-369.

Doma, K., Deakin, G. B., Sealey, R. M., and Leicht, A. S. (2012b). The reliability of running economy among trained distance runners and field-based players. J. Exerc. Sci. Fit., In Press.

Drummond, M. J., Vehrs, P. R., Schaalje, G. B., and Parcell, A. C. (2005). Aerobic and resistance exercise sequence affects excess postexercise oxygen consumption. J. Strength Cond. Res., 19: 332-337.

Faude, O., Meyer, T., Urhausen, A., and Kindermann, W. (2009). Recovery training in cyclists: ergometric, hormonal and psychometric findings. Scand. J. Med. Sci. Sports, 19: 433-441.

Hakkinen, K., Pakarinen, A., Alen, M., Kauhanen, H., and Komi, P. V. (1988). Daily hormonal and neuromuscular responses to intensive strength training in 1 week. Int. J. Sports Med., 9: 422-428.

Hickson, R. C. (1980). Interference of strength development by simultaneously training for strength and endurance. Eur. J. Appl. Physiol. Occup. Physiol., 45: 255-263.

Ide, B. N., Leme, T. C., Lopes, C. R., Moreira, A., Dechechi, C. J., Sarraipa, M. F., Da Mota, G. R., Brenzikofer, R., Macado, D. V. (2011). Time course of strength and power recovery after resistance training with different movement velocities. J. Strength Cond. Res., 25: 2025-2033.

Kellis, E., and Liassou, C. (2009). The effect of selective muscle fatigue on sagittal lower limb kinematics and muscle activity during level running. J. Orthop. Sports Phys. Ther., 39: 210-220.

Leveritt, M., Abernethy, P. J., Bary, B. K., and Logan, P. A. (1999). Concurrent strength and endurance training. Sports Med., 28: 413-427.

Marcora, S. M., and Bosio, A. (2007). Effect of exercise-induced muscle damage on endurance running performance in humans. Scand. J. Med. Sci. Sports, 17: 662-671.

Marshall, P. W., McEwen, M., and Robbins, D. W. (2011). Strength and neuromuscular adaptation following one, four, and eight sets of high intensity resistance exercise in trained males. Eur. J. Appl. Physiol., 111: 3007-3016.

Morgan, D., Daniels, J., Carlson, P., Filarski, K., and Landle, K. (1991). Use of recovery VO2 to predict running economy. Eur. J. Appl. Physiol. Occup. Physiol., 62: 420-423.

Neder, J. A., and Stein, R. (2006). A simplified strategy for the estimation of the exercise ventilatory thresholds. Med. Sci. Sport Exerc., 38: 1007-1013.

Palmer, C. D., and Sleivert, G. G. (2001). Running economy is impaired following a single bout of resistance exercise. J. Sci.
Med. Sport, 4: 447-459.

Paschalis, V., Giakas, G., Baltzopoulos, V., Jamurtas, A. Z., Theoharis, V., Kotzamanidis, C., Koutedakis, Y. (2007). The effects of muscle damage following eccentric exercise on gait biomechanics. Gait Posture., 25: 236-242.

Reeves, M. M., Davies, P. S., Bauer, J., and Battistutta, D. (2004). Reducing the time period of steady state does not affect the accuracy of energy expenditure measurements by indirect calorimetry. J. App. Physiol., 97: 130-134.

Reeves, N. D., Narici, M. V., and Maganaris, C. N. (2003). Strength training alters the viscoelastic properties of tendons in elderly humans. Muscle Nerve., 28: 74-81.

Twist, C., and Eston, R. (2005). The effects of exercise-induced muscle damage on maximal intensity intermittent exercise performance. Eur. J. Appl. Physiol. Occup. Physiol., 94: 652-658.

Winter, D. A. (2008). Biomechanics and motor control of human movement (4th ed.). Hoboken, New Jersey: John Wiley \& Sons, Inc. 


\section{Name:}

Kenji Doma

\section{Affiliation:}

Institute of Sport and Exercise Science, School of Public Health and Tropical Medicine, James Cook University, Australia

\section{Address:}

1 James Cook Drive, Rehabilitation Sciences Building (DB-43), Institute of Sport and Exercise Science, James Cook University, Douglas Campus, Townsville QLD 4811

\section{Brief Biographical History:}

2005-2007: Bachelor of Sport and Exercise Science, James Cook University, Australia

2009-current: Doctrate of Philosophy

2012-current: Research Officer, James Cook University, Australia

\section{Main Works:}

-Doma, K., Deakin, G. B., and Sealey, R. M. (2012). The reliability of lower extremity and thoracic kinematics at various running speeds. International Journal of Sports Medicine, 33(5), 364-369.

-Doma, K., and Deakin, G. B. (2012). The acute effects intensity and volume of strength training on running performance. European Journal of Sports Science, iFirst article, 1-9.

-Doma, K., Deakin, G. B., Leicht, A. S., and Sealey, R. M. (2012). The reliability of running economy among trained distance runners and field-based players. Journal of Exercise Science \& Fitness, 10(2), 90-96.

-Doma, K., and Deakin, G. B. (2013). The effects of strength training and endurance training order on running -economy and -performance. Applied Physiology, Nutrition \& Metabolism, In Press.

-Doma, K., and Deakin, G.B. (2013). Kinematic and electromyographic comparisons between chin-ups and lat-pull down exercises. Sports Biomechanics, In Press.

Membership in Learned Societies:

- Member of Exercise \& Sports Science Australia

- Member of National Strength \& Conditioning Association 